\title{
Commentary \\ Recently published papers: A review of novel strategies in the prevention of hospital-acquired infections, the ability of intensivists to perform echocardiography, and the benefit of polymyxin $B$ haemoperfusion in abdominal sepsis
}

\author{
James Hayward and Richard Venn \\ Department of Anaesthetics, Worthing Hospital, Lyndhurst Road, Worthing BN11 2DH, UK
}

Corresponding author: James Hayward, jnchayward@gmail.com

Published: 26 August 2009

Critical Care 2009, 13:181 (doi:10.1186/cc7992)

This article is online at http://ccforum.com/content/13/4/181

(c) 2009 BioMed Central Ltd

\begin{abstract}
Chlorhexidine bed baths seem to reduce the incidence of methicillin-resistant Staphylococcus aureus and vancomycinresistant enterococcus detected by surveillance cultures. There is also some evidence on the benefit of chlorhexidine mouthwashes in the prevention of ventilator-associated pneumonias. Acidsuppressing drugs increase the incidence of hospital-acquired pneumonias in non-intensive care unit patients, although this association has not been shown in the intensive care setting. Intensivists can be trained to perform basic echocardiography in a short period of time, but their errors could lead to incorrect changes in management. Polymyxin B haemoperfusion was shown in interim analysis to improve patients with abdominal sepsis to such an extent that the EUPHAS randomised controlled trial was halted on ethical grounds, although other authors have criticised this decision.
\end{abstract}

\section{Hospital-acquired infections and ventilator- acquired pneumonias: avoidance strategies}

Prevention of hospital-acquired infections and ventilatorassociated pneumonias is a key concern in the reduction of mortality in both the critical care and general ward patient populations. A number of recent articles have evaluated differing approaches relating to this topic [1-4].

Climo and colleagues studied the effect of daily $4 \%$ chlorhexidine baths on colonisation and subsequent bloodstream infection with multidrug-resistant organisms, in a multicentred before-after study [1]. The acquisition of methicillin-resistant Staphylococcus aureus and vancomycin-resistant enterococcus, as detected by active surveillance cultures, was shown to be significantly reduced (methicillin-resistant $S$. aureus incidence density 5.04 vs. 3.44 cases per 1,000 eligible patient-days, $P=0.046$; vancomycin-resistant enterococcus incidence density 4.35 vs. 2.19 cases per 1,000 eligible patient-days, $P=0.008)$. Furthermore, the authors showed that the incidence of vancomycin-resistant enterococcus bacteremias decreased significantly following the intervention ( 2.13 vs. 0.59 cases per 1,000 patient-days). Owing to the low rate of methicillin-resistant $S$. aureus bacteremia prior to the change in practice, Climo and colleagues were unable to show a significant reduction in methicillin-resistant $S$. aureus bloodstream infections, although they suggest that with sufficiently powered studies this may also be shown to be the case.

Panchabhai and colleagues compared oropharyngeal cleansing using $0.2 \%$ chlorhexidine with the study intensive care unit (ICU) policy of twice-daily $0.01 \%$ potassium permanganate in a randomised controlled trial [2]. Within their study population of 471 patients in total there was no significant difference between groups. The authors do observe, however, that the incidence of nosocomial pneumonias on their ICU in the 3 months prior to the study and in the 3 months after the study was significantly higher than during the 6 -month study period $(21.7 \%$ vs. $7.4 \% ; P<0.001)$. The authors conclude that during the study period the meticulous oral hygiene in both groups and the weak antiseptic properties of potassium permanganate may have contributed to this unanticipated but significant finding. Two other studies have also found that oral decontamination with either chlorhexidine or a chlorhexidine/ collistin mixture did significantly reduce the incidence of ventilator-associated pneumonias $[3,4]$. The National Institute for Health and Clinical Excellence and the National Patient Safety Agency have recommended this practice as a part of the ventilator care bundle since August 2008 [5].

In the management of severe sepsis or septic shock, the Surviving Sepsis Campaign [6] strongly recommends the use of a histamine-2 blocker or a proton-pump inhibitor in order to 
provide stress ulcer prophylaxis. Herzig and colleagues undertook a large single-centred cohort study of the effect of acid-suppressing medications on the incidence of hospitalacquired pneumonias [7]. They included all patients admitted to their hospital for longer than 3 days over a period of 3 years $(n=42,093)$, excluding patients admitted to the ICU. Of these patients, acid-suppressing medication was ordered in 32,933 (52\%) admissions. There was a large baseline difference in the characteristics of the group receiving acidsuppressing medications versus those patients not receiving the medications. Once these groups had been propensity matched, the authors were able to compare 16,396 paired patients and showed that the use of an acid-suppressing drug showed an increased association with developing a hospital-acquired pneumonia with an odds ratio of 1.3 (95\% confidence interval $=1.1$ to 1.4 ). However Beaulieu and colleagues in their 2008 study did not show an increased risk of nosocomial pneumonia in medical intensive care patients receiving proton-pump inhibitors [8]. Given these findings, the prescription of acid-suppressing medications should be given careful consideration - particularly when used for the purpose of stress ulcer prophylaxis, where these drugs should be ceased as soon as enteral feeding has been reestablished.

Schweickert and colleagues present the argument that early physical and occupational therapy can reduce the duration of time on a ventilator, and can thereby reduce the mortality associated with this intervention [9]. They performed a dualcentre randomised controlled trial comparing standard ICU practice of daily sedation holds and therapy as per the primary care team versus daily sedation holds with specific early exercise and mobilisation (physical and occupational therapy). In their group of 104 patients, early physical and occupational therapy significantly increased the number of patients who had returned to independent functional status by the point of discharge (59\% vs. 35\%, $P=0.02)$. Early therapy also was shown to reduce the average number of days with ICU delirium (2.0 vs. 4.0, $P=0.03$ ). Early therapy did not, however, reduce the duration of ICU stay (5.9 vs. 7.9, $P=0.08)$ or of hospital stay (13.5 vs. $12.9, P=0.93$ ).

Knight and colleagues looked at a less commonly described intervention, enteral synbiotic (a mixture of prebiotic and probiotic) therapy, on the incidence of ventilator-associated pneumonias [10]. In their blinded, randomised, placebocontrolled trial, the authors compared a total of 259 patients with a primary outcome variable as the development of ventilator-acquired pneumonia. The use of synbiotics had no significant effect on the development of ventilator-acquired pneumonia, nor the secondary endpoints of oropharyngeal flora, ventilator days, ventilator-associated pneumonia rate per 1,000 ventilated days, ICU length of stay and hospital length of stay.

\section{Intensivist assessment of left-ventricular function using transthoracic ultrasound}

To appropriately manage the critically ill patient, an accurate assessment of left ventricular function is often called for. Owing to equipment or personnel limitations these investigations can be significantly delayed, and a rapid assessment of left ventricular function is often not possible.

Melamed and colleagues asked whether, after brief training, intensivists were able to make an accurate assessment of left ventricular function using transthoracic ultrasound [11]. They trained a group of intensivists with 2 hours of didactic teaching and 4 hours of practical teaching, and provided a range of pre-recorded examinations for the intensivists to study in their own time. The study group comprised 44 patients in whom the intensivists were asked to first identify normal or abnormal left ventricular function and then to categorise those with abnormal function into mild to moderate or severe. Ultimately, the intensivists correctly categorised 36 out of 44 cases (82\%). It would be easy to conclude that this is a resounding endorsement for transthoracic echocardiography performed by the intensivist, but the cases they incorrectly identified were overestimations of actual function and this could potentially alter management incorrectly. If the study had also evaluated the degree of certainty that the intensivists held over their findings, it would help to illustrate whether inappropriate changes of management might have resulted from their transthoracic echocardiography findings.

\section{Effect of polymyxin B haemoperfusion on patients with abdominal sepsis}

The EUPHAS randomised controlled trial examined the effect of polymyxin $B$ fibre haemoperfusion on the outcome of patients with abdominal septic shock [12]. Polymyxin $B$ is an antibiotic with a high affinity for endotoxin. Haemoperfusion was performed using haemoperfusion polystyrene fibres with bound polymyxin B. The study was stopped early because there was a signal of decreased mortality in the treatment group, based on a Cox proportional hazards regression survival model. The crude mortality analysis, however, did not show a statistically significant difference between groups (11/34 polymyxin group vs. $16 / 30$ conventional group, odds ratio $=2.39(95 \%$ confidence interval $=0.87$ to 6.60$)$, $P=0.09$ ). The difference between the crude mortality analysis and the Cox regression model can be explained by a larger occurrence of late deaths in the treated group. This is a concerning observation in an unblinded trial, as it could be caused by a brief prolongation of life support in the treatment group and a resultant increase in early survival data. Despite their early withdrawal from the study the authors call for larger multicentred studies to confirm their findings. The decision for early cessation, the study's insufficient power and the lack of a patient-centred endpoint has been criticised in other editorials [13]. 


\section{Competing interests}

The authors declare that they have no competing interests.

\section{References}

1. Climo MW, Sepkowitz KA, Zuccotti G, Fraser VJ, Warren DK, Perl TM, Speck K, Jernigan JA, Robles JR, Wong ES: The effect of daily bathing with chlorhexidine on the acquisition of methicillin-resistant Staphylococcus aureus, vancomycin-resistant enterococcus and healthcare-associated bloodstream infections: results of a quasi-experimental multicenter trial. Crit Care Med 2009, 37:1858-1865.

2. Panchabhai TS, Dangayach NS, Krishnan A, Kothari VM, Karnad DR: Oropharyngeal cleansing with $0.2 \%$ chlorhexidine for prevention of nosocomial pneumonia in critically ill patients. Chest 2009, 135:1150-1156.

3. Koeman M, van der Ven AJ, Hak E, Joore HC, Kaasjager K, de Smet AG, Ramsay G, Dormans TP, Aarts LP, de Bel EE, Hustinx WN, van der Tweel I, Hoepelman AM, Bonten MJ: Oral decontamination with chlorhexidine reduces the incidence of ventilator-associated pneumonia. Am J Respir Crit Care Med 2006, 173:1348-1355.

4. Tantipong $\mathrm{H}$, Morkchareonpong $\mathrm{C}$, Jaiyindee $\mathrm{S}$, Thamlikitkul V: Randomised controlled trial and meta-analysis of oral decontamination with $2 \%$ chlorhexidine solution for the prevention of ventilator-associated pneumonia. Infect Control Hosp Epidemio/ 2008, 29:131-136.

5. National Institute for Health and Clinical Excellence/National Patient Safety Agency: Technical Patient Safety Solutions for Ventilator-associated Pneumonia in Adults. London: NICE; 2008.

6. Dellinger RP, Levy MM, Carlet JM, Bion J, Parker MM, Jaeschke R, Reinhart K, Angus DC, Brun-Buisson C, Beale R, Calandra T, Dhainaut JF, Gerlach H, Harvey M, Marini JJ, Marshall J, Ranieri M, Ramsay G, Sevransky J, Thompson BT, Townsend S, Vender JS, Zimmerman JL, Vincent JL: Surviving Sepsis Campaign: international guidelines for management of severe sepsis and septic shock: 2008 [special article]. Intensive Care Med 2008, 34:1760.

7. Herzig SJ, Howell MD, Ngo LH, Marcantonio ER: Acid-suppressive medication use and the risk-for hospital acquired pneumonias. JAMA 2009, 301:2120-2128.

8. Beaulieu M, Williamson D, Sirois C, Lachaine J: Do proton-pump inhibitors increase the risk for nosocomial pneumonia in a medical intensive care unit? J Crit Care 2008, 23:513-518.

9. Schweickert WD, Pohlman MC, Pohlman AS, Nigos C, Pawlik AJ, Esbrook CL, Spears L, Miller M, Franczyk M, Deprizio D, Schmidt GA, Bowman A, Barr R, McCallister KE, Hall JB, Kress JP: Early physical and occupational therapy in mechanically ventilated, critically ill patients: a randomised controlled trial. The Lancet 2009, 373:1874-1882.

10. Knight DJ, Gardiner D, Banks A, Snape SE, Weston VC, Bengmark S, Girling KJ: Effect of synbiotic therapy on the incidence of ventilator associated pneumonia in critically ill patients: a randomised, double-blind, placebo-controlled trial. Intensive Care Med 2009, 35:854-861.

11. Melamed R, Sprenkle MD, Ulstad VK, Herzog CA, Leatherman JW: Assessment of left ventricular function by intensivists using hand-held echocardiography. Chest 2009, 135:14161419.

12. Cruz DN, Antonelli M, Fumagalli R, Foltran F, Brienza N, Donati A, Malcangi V, Petrini F, Volta G, Bobbio Pallavicini FM, Rottoli F, Giunta F, Ronco C: Early use of polymyxin B hemoperfusion in abdominal septic shock. The EUPHAS randomised controlled trial. JAMA 2009, 301:2445-2452.

13. Kellum JA, Uchino S: International differences in the treatment of sepsis. Are they justified? JAMA 2009, 301:2496-2497. 